\title{
Renate Künast MdB
}

\section{Bundesministerin für Verbraucherschutz, Ernährung und Landwirtschaft a.D.}

\section{Vorsitzende der Bundestagsfraktion Bündnis 90/Die Grünen}

\author{
Juristinnen machen Karriere - wir stellen sie vor
}

Die Fragen stellten Maria Wersig, Mitglied der Kommission Recht der sozialen Sicherung, Familienlastenausgleich des djb, und Barbara Dittmann, Vertreterin der Mitglieder in Ausbildung im Bundesvorstand von 2005 bis 2009 , im Juni 2010.

Warum haben Sie nach dem Abschluss des Studiums der Sozialarbeit und mehrjähriger Berufstätigkeit noch Jura studiert? War Rechtsanwältin damals - zu Beginn des Studiums - ihr Ziel?

Ich habe als Sozialarbeiterin gelernt, dass man sehr viel erreichen kann mit persönlichem Einsatz für eine wichtige Sache. Um aber an den Strukturen etwas zu verändern, braucht es andere Werkzeuge. Dafür ist ein Studium der Rechtswissenschaften sehr gut. Klar war mir von Beginn an, dass ich dazu beitragen wollte, die Verhältnisse zu ändern. Da kommt frau an den Rechtswissenschaften nicht vorbei.

Hat Ihre Familie und Ihr weiteres persönliches Umfeld Sie in Ihrem Berufs(wechsel)wunsch unterstützt?

Es gab immer Menschen in meinem Leben, die mich unterstützt haben. In der Grundschule war es meine Lehrerin, die meinem Vater sagte: Die kann und will etwas, sie soll noch weiterlernen.

Was würden Sie heute anders machen, wenn Sie noch einmal am Anfang Ihrer Karriere stünden? Würden Sie überhaupt etwas anders machen?

Wenn ich heute rückwärts schaue, hätte ich mich früher und stärker mit Sprachen und Wirtschaftspolitik beschäftigt. Aber was soll's - ich habe jeweils gemacht, was ich mir zutraute und was mich reizte.

Hatten Sie zu Beginn Ihrer Karriere weibliche Vorbilder? Haben Sie (dieselben) auch heute noch? Wenn ja, was macht deren Vorbildcharakter für Sie aus?

Für Frauen gab es in den Achtzigerjahren noch nicht viele weibliche Vorbilder in Politik, Wirtschaft und Wissenschaft. Schon als Teeny fand ich Hildegard Hamm-Brücher beeindruckend. Heute auch noch. Sie ist selbstbewusst und gradlinig.

Heute bewundere ich die vielen Heldinnen des Alltags. Frauen, die jeden Tag neu die Doppel- oder Dreifachbelastung meistern. Sie schaffen ihren Job - oft genug schlecht bezahlt als Altenpflegerin, Verkäuferin, Friseurin und schaffen es, auch noch die Erziehung und Betreuung von Kindern unter einen
Renate Künast, geboren am 15. Dezember 1955 in Recklinghausen/ NRW, studierte zunächst Sozialarbeit an der Fachhochschule in Düsseldorf und arbeitete von 1977 bis 1979 als Sozialarbeiterin in der Justizvollzugsanstalt Berlin-Tegel, speziell mit Drogenabhängigen. Später studierte sie Jura und schloss das Studium 1985 mit dem zweiten Staatsexamen ab. Sie ist Rechtsanwältin.

Der Westberliner Alternativen Liste trat sie 1979 bei und hat seitdem in verschiedenen Funktionen für die Partei gearbeitet. Während der rot/grünen Koalition in Berlin in den Jahren 1989/90 war sie Fraktionsvorsitzende. Nach dem Ende des rot/grünen Senats arbeitete sie weiter als Abgeordnete in der Fraktion Bündnis 9o/Die Grünen, bis 1993 und 1998 bis 2000 als deren Vorsitzende, dazwischen als rechtspolitische Sprecherin. Renate Künast war von Juni 2000 bis März 2001 Bundesvorsitzende von Bündnis 9o/Die Grünen. Sie war von Januar 2001 bis zum 4. Oktober 2005 Bundesministerin für Verbraucherschutz, Ernährung und Landwirtschaft.

Seit dem 18. Oktober 2005 ist sie Fraktionsvorsitzende der Bundestagsfraktion von Bündnis 9o/Die Grünen.

Hut zu bekommen. Was sie leisten, grenzt häufig an das menschlich mögliche. Sie brauchen bessere Bezahlung, bessere Betreuung und oft genug auch emanzipiertere Männer, was die Beteiligung an den Haushalts- und Erziehungspflichten angeht.

Ist es Ihrer Meinung nach wichtig, dass sich junge Frauen bzw. junge Akademikerinnen an weiblichen Rollenvorbildern orientieren und mit ihnen im gegenseitigen Austausch stehen, oder haben Sie vielleicht die Erfahrung gemacht, dass auch männliche Rollenvorbilder zur persönlichen und beruflichen Weiterentwicklung geeignet sind?

Frauen müssen sich noch viel mehr gegenseitig fördern als es bisher geschieht. Männer sind richtig gut darin, sich gegenseitig Informationen, Tipps und gute Jobs zuzuschachern und manche kämpfen für den Erhalt reiner Männerherrlichkeit in den Vorstandsetagen. Wir Frauen müssen mehr Netzwerke bauen. Keine Scheu: es ist hochprofessionell, Reden mit Kolleginnen oder Freundinnen vorzubereiten. Oder Frauen bei Bewerbungen zu unterstützen. Und wenn einzelne Männer Frau- 
en fördern, ist das ebenso gut, wenn sie damit junge Frauen auf ihrem Weg unterstützen.

Sie sind Berufspolitikerin und führen mit Kollegen zusammen eine Kanzlei, sind also auch als Rechtsanwältin tätig. Nebenher engagieren Sie sich vielseitig ehrenamtlich. Sind Sie es schon aus Schulzeiten gewohnt, sich stark zu engagieren und Ihre Zeit und Ihr Engagement auf viele Bereiche aufzuteilen?

Langeweile war mir schon als Kind ein Graus. Auch den großen inneren Wunsch, etwas zu verändern, zu gestalten, hatte ich schon als junge Frau in mir. Ich achte darauf, genügend Zeit für meine Leidenschaften zu haben. Kochen, im Garten arbeiten, gute Bücher lesen. Das ist das notwendige kreative Ausspannen. entwickeln. Alte, fast ausgestorbene Sorten anzupflanzen und so ganz praktisch Artenvielfalt zu betreiben, das finde ich schön. In Stressphasen nehme ich mir die Zeit für Saunabesuche, Massage und Shiatsu. Sich nur auf sich selbst zu konzentrieren ist regelmäßig nötig.

Ist es in der Politik schwieriger, gleichstellungspolitische Themen auf die Agenda zu setzen als zum Beispiel umweltpolitische?

Umweltpolitik und Gleichstellungspolitik haben gemeinsam, dass sie nicht von Beginn an als zentrale gesellschaftliche Herausforderungen verstanden wurden. Das hat sich bei der Umweltpolitik mittlerweile geändert. Für Gleichstellungspolitik gilt dies leider noch nicht. Das sehen Sie an der leidigen Debatte um die Quotierung von Aufsichtsräten. Wo sonst gibt es in

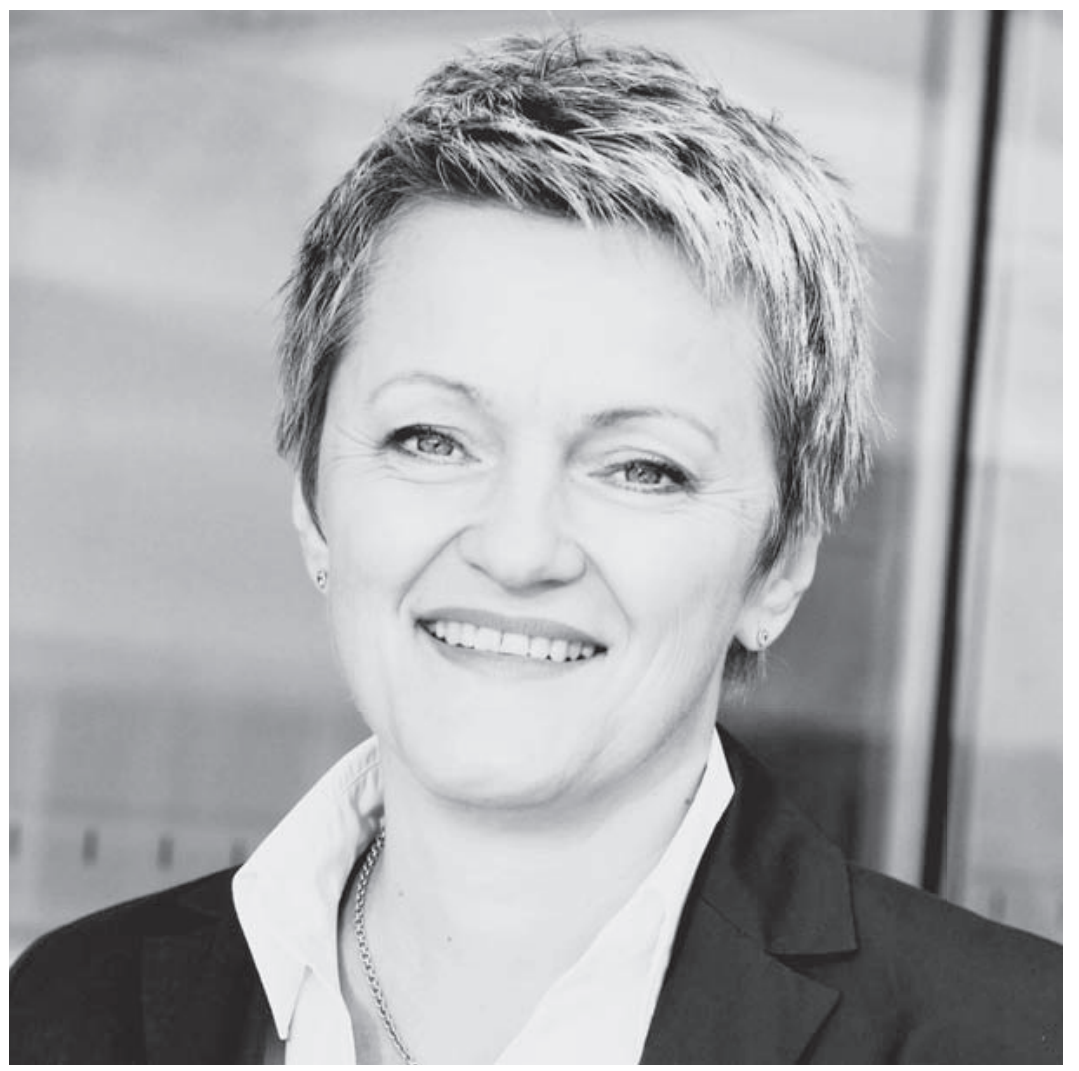

Können Sie unseren jungen Juristinnen Anregungen geben, wie sich so viele Aktivitäten unter einen Hut bringen lassen? Wer eine Führungsposition hat, muss lernen zu delegieren und ein gutes Zeitmanagement haben. Als Erstes im Kalender Zeiträume blocken. Für Ferien, für Wochenenden, Abende mit Freunden und auch das individuelle Ausspannen. Der ganze Rest unterliegt einem straffen Management und der Bereitschaft, die Wichtigkeit eines Termins wieder anzuzweifeln.

\section{Wie entspannen Sie?}

Meine großen Leidenschaften sind Kochen und Gärtnern. Ich empfinde es als Wohltat zu sehen, wie Dinge wachsen und sich der Politik eine solche Blindheit gegenüber den Fakten? Zehn Jahre schon gibt es die freiwillige Selbstvereinbarung mit der Wirtschaft für mehr Gleichstellung. Obwohl der Frauenanteil kaum gestiegen ist, gibt sich die Mehrheit der Konservativen uneinsichtig. Wir bringen als Flaggschiff jetzt einen Aufsichtsratsantrag im Bundestag ein. Alle Leserinnen sind herzlich eingeladen, das massiv zu unterstützen.

Sie haben als Ministerin dem Ressort Landwirtschaft den Verbraucherschutz hinzugefügt. Warum engagieren Sie sich hier besonders stark?

Ich bin überzeugt, dass Verbraucher eben auch Rechte haben im Wirtschaftsleben. $\mathrm{Zu}$ wissen, was drin ist, rechtliches 
Gehör zu bekommen. Und vor allem: mit dem Einkaufskorb Politik zu machen.

Welche Einflussmöglichkeiten hat Politik Ihrer Meinung nach auf den privaten Verbraucher, tragen etwa Ampeln auf Nahrungsmittelverpackungen nachweislich zu gesünderem Essverhalten bei? Kann Regulierung den uninteressierten Verbraucher überzeugen bzw. „erziehen“? Welches ist Ihrer Überzeugung nach das Verbraucherleitbild hierzulande beziehungsweise in Europa und steht staatliche Regulierung nicht dem Leitbild des mündigen Verbrauchers entgegen?

Ich will gut informierte Verbraucher! Nur so können sie eine bewusste und freie Entscheidung treffen. Es ist Aufgabe des Staates, hier den richtigen Ordnungsrahmen zu setzen und diese Verbraucherrechte zu stärken. Transparenz ist das Gebot und saubere Lebensmittel sowie fair hergestellte Produkte. Die letzte Entscheidung trifft der Verbraucher selbst.

Was war bisher Ihr größter politischer Erfolg und was wollen Sie noch erreichen?

Mein Traum ist ein Europa, das wirklich nachhaltig lebt und wirtschaftet. Vom Biosiegel über bessere Lebensmittel bis zu erneuerbarer Energie habe ich an ein paar guten Sachen mitgearbeitet.

Sie haben als Parteimitglied von Bündnis9o/Die Grünen lange Zeit eine Partei erlebt, die in ihrer Außenwirkung als im Schatten eines patriarchalischen Parteivorsitzenden Joschka Fischer stehend erschien. Später standen Sie mit Fritz Kuhn an der Doppelspitze Ihrer Partei. Welche Erfahrungen haben Sie als Frau mit Führungsrollen in der Politik, aktiv wie passiv, gemacht?

Geschlechtergerechtigkeit ist nirgendwo eine Selbstverständlichkeit. Wir grüne Frauen haben sie uns mit der Quote erkämpft. Die schwierigsten Zeiten hatte ich sowohl in der Juristerei, als auch in der Politik in den Anfängen; als die zum Beispiel in Parlamentsausschüssen vorherrschende fast 100-Prozent-Männerquote schon durch die Anwesenheit einer Frau infrage gestellt war. Die Überheblichkeit, die einem entgegenschlug, diese klassischen Männerwitze machten die Arbeit nicht gerade zu einem Vergnügen.

Sind bei Bündnis 9o/Die Grünen die Frauen in den Führungspositionen samt und sonders sogenannte "Quotenfrauen"? Also sind auch Sie eine „Quotenfrau“?

Ja, ich bin eine Quotenfrau. Aber in den Vorstandsetagen sitzen auch viele Männer, die ihre Jobs nur kriegten, weil es eine 100-Prozent-Männerquote gab oder gibt.
Was tun Sie als Fraktionsvorsitzende für die Förderung von Frauen in der Politik?

Ich selbst fördere verschiedene Frauen, tausche mich mit ihnen aus und gebe Tipps. Gerade habe ich wieder eine MentoringPartnerschaft mit einer jungen Frau begonnen. Der Austausch ist auch für mich spannend. Innerhalb der Fraktion gibt es verschiedene Ebenen, auf denen die weiblichen Abgeordneten vernetzt sind.

Gibt es einen männlichen und einen davon abweichenden weiblichen Führungsstil?

In der Politik waren für Frauen lange keine Rollen vorgesehen. Das hat zuerst einmal geheißen, dass Frauen sich den „männlichen Werkzeugkoffer“ aneignen mussten. Egal ob Kleidungsstil, Verhandlungsrituale oder das Netzwerken. Die gesetzte Norm war männlich. Heute differenziert es sich langsam. Aber eben nur langsam. Die öffentliche Vorstellung eines Alpha-Tieres hat fast nur männliche Attribute.

Welche Tipps haben Sie für junge Frauen, die in Männerdomänen wie den Führungsetagen der Politik, Justiz oder auch der Wirtschaft Erfolg haben wollen? Welche besonderen Fallen gilt es zu umgehen?

Sie sollen mutig sein und zeigen: „Hier bin ich, das kann ich und das will ich“. Und sie sollten sich unbedingt gut vernetzen mit anderen Frauen und Männern. Nach wie vor sind viele Frauen zu selbstkritisch. Ich glaube, uns Frauen tut ein bisschen Gelassenheit gut. Stolz sein auf Erfolge und Niederlagen nicht allzu persönlich zu nehmen, dass können die meisten Männer viel besser.

Für wie wichtig erachten Sie speziell im juristischen und im politischen Berufsumfeld fachspezifische beziehungsweise allgemein Frauennetzwerke?

Netzwerke sind überaus wichtig. Sowohl für den fachlichen Austausch als auch für alles Informelle. Aber sie müssen klar definierte Ziele haben. Nur gemeinsam vereinbarte Ziele und entsprechendes Handeln führen zum Erfolg.

Sie sind Mitglied des djb, was uns sehr freut. Wenn Sie heute eine junge Juristin auf den djb ansprechen würde, welche Gründe für einen Beitritt zu unserem Verband würden Sie ihr nennen?

Gib anderen von deiner Stärke ab, erhalte Stärke zurück. Nutze den Rückhalt und auch die Schubkraft, die im Gemeinsamen liegt. Hört sich pathetisch an, ist aber treffend! 